\title{
LOCALIZABLE ANALYTICALLY UNIFORM SPACES AND THE FUNDAMENTAL PRINCIPLE
}

BY

\author{
SÖNKE HANSEN
}

\begin{abstract}
The Fundamental Principle of Ehrenpreis states that the solutions of homogeneous linear partial differential equations with constant coefficients have natural integral representations. Using the Oka-Cartan procedure Ehrenpreis derived this theorem for spaces of functions and distributions which he called localizable analytically uniform (LAU-spaces). With a new definition of LAUspaces we explain how Hörmander's results on cohomology with bounds fit into Ehrenpreis' method of proof of the Fundamental Principle. Furthermore, we show that many of the common Fréchet-Montel spaces of functions are LAU-spaces.
\end{abstract}

1. Introduction. Let $\Omega \subset \mathbf{R}^{n}$ be open and convex and denote by $\mathcal{E}(\Omega)$ the space of infinitely differentiable functions in $\Omega$ with its usual Schwartz topology. Let $P(D)=\left(P_{l k}(D)\right)$ be an $L \times K$-matrix of differential operators with constant coefficients (where $D=-i \partial / \partial x$ ). Let $u=\left(u_{1}, \ldots, u_{k}\right) \in \mathcal{E}(\Omega)^{K}$. Then the Fundamental Principle proved by Ehrenpreis [8], [9] and, independently, by Palamodov [16] states that $u$ satisfies the homogeneous equation $P(D) u=0$ if and only if a representation

$$
u_{k}(x)=\sum_{1}^{J} \int_{V_{j}} d_{j k}\left(z, \partial_{z}\right) e^{i\langle z, x\rangle} d \mu_{j}(z)
$$

holds for all $x \in \Omega$ and $k=1, \ldots, K$. Here $J$ is a positive integer, $\left(d_{j}\right)=$ $\left(d_{j k}\left(z, \partial_{z}\right)\right)$ is a $J \times K$-matrix of differential operators with polynomial coefficients and the $V_{j}$ 's are algebraic varieties contained in the characteristic variety $V$ for $P(D)$;

$$
V=\left\{z \in \mathbf{C}^{n} ; \operatorname{ker} P(z) \neq 0\right\} .
$$

$P(z)$ is considered here as a linear map from $\mathbf{C}^{K}$ into $\mathbf{C}^{L}$. The operators $d_{j k}$ and the varieties $V_{j}$ only depend on $P(D)$. The Radon measures $d \mu_{j}$ have supports contained in $V_{j}$ and satisfy, with a suitable positive continuous function $k$ on $\mathbf{C}^{n}$, the growth condition

$$
\int_{V_{j}} k(z)\left|d \mu_{j}\right|(z)<+\infty
$$

which is such that it insures the convergence of the integrals (1.1) for every $x \in \Omega$. More precisely, (1.2) holds for some member $k$ of the family $\mathcal{K}$ of all positive continuous functions $k$ on $\mathbf{C}^{n}$ such that

$$
\sup _{z \in \mathbf{C}^{n}}|\hat{\varphi}(z)| / k(z)<+\infty
$$

Received by the editors October 29, 1979 and, in revised form, February 15, 1980.

1980 Mathematics Subject Classification. Primary 46E10, 46F05, $35 \mathrm{C15}$. 
for every $\varphi \in \mathcal{E}^{\prime}(\Omega)$ (here $\hat{\varphi}(z)=\varphi\left(e^{-i\langle;, z\rangle}\right), z \in \mathbf{C}^{n}$, is the Fourier-Laplace transform). It turns out that the family $\mathcal{K}$ defines a seminorm topology on $\mathcal{E}^{\prime}(\Omega)$ which coincides with the usual strong topology ( $\Omega$ is assumed to be convex). The seminorms are given by the numbers (1.3). $\mathscr{K}$ is called an analytically uniform structure for $\mathscr{E}(\Omega)$. Its importance lies in the fact that it enables one to find measures $d \mu_{j}$ such that (1.1) is valid for all $x \in \Omega$ whereas the Paley-Wiener estimates would a priori only lead to a representation (1.1) valid for all $x$ in an arbitrary but fixed compact subset of $\Omega$, i.e. the measures $d \mu_{j}$ would also depend on this compact.

For the proof of the Fundamental Principle it is essential that the analytically uniform structure $\mathcal{K}$ satisfies an additional condition called localizability of Ehrenpreis. Let us briefly indicate the reason for this. The kernel of $P(D)$ is via the Fourier-Laplace transform canonically dual to a quotient space of a locally convex space of entire functions $f$ with seminorms $\sup _{z \in \mathbf{C}^{\mathbf{c}}}|f(z)| / k(z)<+\infty, k \in \mathcal{K}$. The proof of the Fundamental Principle therefore rests on a description of this quotient space. More specifically, one has to find in every coset a representative satisfying good bounds (stemming from $\mathcal{K}$ ). Such a representative $f$ can be given by first constructing it locally and afterwards globally on $\mathbf{C}^{n}$ using a suitable theory of cohomology with bounds. The localizability condition on $\mathscr{K}$ is then introduced to insure that the bounds given by $\mathcal{K}$ are compatible with the bounds necessitated by the cohomology.

In this paper we give a treatment of the more functional analytic part of the proof of the Fundamental Principle. Following Ehrenpreis' approach outlined above we do this for linear partial differential equations with constant coefficients in localizable analytically uniform spaces (LAU-spaces). The LAU-spaces which we use are closely related to but more general than Ehrenpreis' PLAU-spaces. The main point is that the localizability condition which we impose is well suited for working with Hörmander's results on cohomology with bounds (see [12, Chapter 7.6]), while Ehrenpreis' condition enables one to use a quantitative version of the Oka-Cartan procedure. So we in particular clarify the relationship between Ehrenpreis' method and Hörmander's results. This task was suggested to us by a remark made by Ehrenpreis in his book (see [9, Remark 1.6]). We introduce localizable analytically uniform structures in $\$ 2$. In $\$ 3$ the Fundamental Principle is derived for LAU-spaces from a theorem on division and extension of entire functions with bounds. That theorem will be stated without proof. We should however mention that its proof contains the hardest parts of the proof of the Fundamental Principle. In $\$ 4$ we give examples of LAU-spaces. In particular $\mathcal{E}(\Omega)$, $\Omega \subset \mathbf{R}^{n}$ open and convex, and similar spaces of ultradifferentiable functions are shown to be LAU-spaces.

2. Localizable analytically uniform structures. Let $\mathcal{K}$ be a nonempty set of positive continuous functions on $\mathbf{C}^{n}$. For $k \in \mathcal{K}$ and every complex valued function $f$ on $\mathbf{C}^{n}$ define

$$
p_{k}(f)=\sup _{z \in \mathbf{C}^{n}}|f(z)| / k(z) .
$$


This is a nonnegative number or $+\infty$. Let $A_{\mathscr{K}}$ (resp. $C_{\mathscr{K}}$ ) denote the locally convex space of all entire (resp. continuous complex valued) functions $f$ on $\mathbf{C}^{\text {n }}$ with $|f(z)| / k(z) \rightarrow 0$ as $z \rightarrow \infty$ for every $k \in \mathscr{K}$ and the topology given by the seminorms $p_{k}, k \in \mathscr{K}$. Let $\mathscr{K}=\mathscr{N}(\mathcal{K})$ be the set of all nonnegative upper semicontinuous functions $m$ on $\mathbf{C}^{n}$ such that $m / k$ is bounded on $\mathbf{C}^{n}$ for every $k \in \mathscr{K}$. The set $\mathscr{N}$ describes the bounded sets in $A_{\mathscr{K}}$ and in $C_{\mathscr{K}}$. In fact, for every bounded set $B$ in $C_{\mathscr{K}}$ there is $m \in \mathscr{T}_{\mathcal{L}}$ with $B \subset\left\{f \in C_{\mathscr{K}} ;|f(z)|<m(z), z \in \mathbf{C}^{n}\right\}$. Now consider the following two conditions on $\mathscr{K}$.

(AU) For every $k \in \mathscr{K}$ there exists $k^{\prime} \in \mathscr{K}$ with

$$
k^{\prime}\left(z+z^{\prime}\right) \cdot\left(2+|z|^{2}\right)<k(z)
$$

for all $z, z^{\prime} \in \mathbf{C}^{n}$ with $\left|z^{\prime}\right| \leqslant 1$.

(L) For every $k \in \mathscr{K}$ there exists $k^{\prime} \in \mathscr{K}$ such that one can find for every $m \in \mathfrak{N}(\mathscr{K})$ with $m(z) \leqslant k^{\prime}(z), z \in \mathbf{C}^{n}$, a plurisubharmonic function $\varphi$ on $\mathbf{C}^{n}$ with $e^{\varphi} \in \mathscr{R}(\mathcal{K})$ and

$$
m(z) \leqslant e^{\varphi(z)} \leqslant k(z), \quad z \in \mathbf{C}^{n} .
$$

Definition 1. Let $\mathscr{K}$ be a nonempty set of positive continuous functions on $\mathbf{C}^{\boldsymbol{n}}$. $\mathcal{K}$ is called an analytically uniform structure (= AU-structure) iff it satisfies (AU). $\mathscr{K}$ is called a localizable analytically uniform structure (= LAU-structure) iff it satisfies (AU) and (L).

Recall that a function $\varphi: \mathbf{C}^{n} \rightarrow[-\infty,+\infty)$ is plurisubharmonic iff it is upper semicontinuous and its restriction to every complex one-dimensional line is subharmonic. Besides other well-known properties of plurisubharmonic functions to which we refer ([11] and [12]) we shall frequently make use of the following fact: The smallest upper semicontinuous majorant of the supremum of a sequence of plurisubharmonic functions is also a plurisubharmonic function provided this supremum is uniformly bounded from above on every compact subset of $\mathbf{C}^{\boldsymbol{n}}$. This follows immediately from Lemma 3.3 in [11] if one expresses the sup by a suitable lim sup.

REMARK. Ehrenpreis calls a subset $\mathscr{T}_{1} \subset \mathfrak{T}(\mathscr{K})$ a sufficient bounded analytically uniform structure (= BAU-structure) for $\mathscr{K}$ if for every $k \in \mathscr{K}$ there exists $k^{\prime} \in \mathscr{K}$ such that one can find for every $m \in \mathscr{N}(\mathscr{K})$ with $m(z)<k^{\prime}(z), z \in \mathbf{C}^{n}$, an element $m^{\prime} \in \mathscr{T}_{1}$ with $m(z) \leqslant m^{\prime}(z) \leqslant k(z), z \in \mathbf{C}^{n}$. So condition (L) just means that the set of all logarithmically plurisubharmonic functions in $\mathfrak{N}$ is a sufficient BAU-structure for $\mathcal{K}$. Note that in (L) one only needs to consider all $\boldsymbol{m}$ belonging to a sufficient BAU-structure. It is therefore convenient to know that the subset of all continuous functions in $\mathscr{T}(\mathcal{K})$ is sufficient if $\mathscr{K}$ is an AU-structure. To prove this just set with some fixed partition of unity $\left(\alpha_{j}\right)_{1}^{\infty}$ with uniform bound $N>0$ on the number of overlaps and on the diameters of the supports for a given $m \in \mathfrak{K}(\mathscr{K})$

$$
m^{\prime}(z)=\sum_{1}^{\infty} \sup _{\alpha_{j}\left(z^{\prime}\right) \neq 0} m\left(z^{\prime}\right) \alpha_{j}(z), \quad z \in \mathbf{C}^{n}
$$


Then $m^{\prime}$ is continuous and $m(z) \leqslant m^{\prime}(z) \leqslant N \cdot \sup \left\{m\left(z+z^{\prime}\right) ;\left|z^{\prime}\right|<N\right\}, z \in \mathbf{C}^{n}$. The statement now follows easily from (AU).

Let us collect some basic properties of the spaces $A_{\mathscr{K}}$.

Proposition 1. Let $\mathcal{K}$ be an $A U$-structure. Then $A_{\mathscr{K}}$ is closed under translations, differentiations and under multiplication by polynomials, and these operators are continuous on $A_{\mathscr{K}} . T$ is a continuous linear functional on $A_{\mathscr{K}}$ iff there exists $a$ (nonunique) Radon measure $d \mu$ on $\mathbf{C}^{n}$ with $\int k(z)|d \mu|(z)<+\infty$ for some $k \in \mathcal{K}$ and

$$
T(f)=\int_{\mathbf{C}^{n}} f(z) d \mu(z) \text { for all } f \in A_{\mathscr{K}} .
$$

If $\mathscr{K}$ is a LAU-structure there exists for every $z_{0} \in \mathbf{C}^{n}$ a function $f \in A_{\mathscr{K}}$ with $f\left(z_{0}\right)=1$.

Proof. The first part of the proposition follows from a repeated application of (AU).

A continuous linear functional $T$ on $A_{\mathscr{K}}$ can be extended for some $k \in \mathcal{K}$ by the Hahn-Banach theorem to the Banach space of all continuous functions $f$ on $\mathbf{C}^{n}$ with $f(z) / k(z) \rightarrow 0$ as $z \rightarrow \infty$ and the norm $p_{k}$. The assertion on $T$ then follows from the Riesz representation theorem.

Now assume that $\mathscr{K}$ is a LAU-structure. Since $\mathscr{T}$ contains all nonnegative continuous functions with compact support we can find by (L) a plurisubharmonic function $\varphi$ with $e^{\varphi} \in \mathfrak{N}$ such that $e^{-\varphi}$ is integrable over a neighbourhood of zero. By Theorem 4.4.4 in [12] there exists an entire function $f$ with $f(0)=1$ and

$$
\int|f(z)|^{2} e^{-2 \varphi(z)}\left(1+|z|^{2}\right)^{-3 n} d \lambda(z)=a^{2}<+\infty .
$$

Here $d \lambda$ denotes the Lebesgue measure on $\mathbf{C}^{n}$. We can pass from the $L^{2}$ - to the sup-estimate:

$$
\begin{aligned}
|f(z)| & \leqslant c_{n}\left(\int_{\left|z^{\prime}\right|<1}\left|f\left(z+z^{\prime}\right)\right|^{2} d \lambda\left(z^{\prime}\right)\right)^{1 / 2} \\
& \leqslant c_{n} a \cdot \sup _{\left|z^{\prime}\right|<1} e^{\varphi\left(z+z^{\prime}\right)}\left(1+\left|z+z^{\prime}\right|^{2}\right)^{3 n / 2}
\end{aligned}
$$

holds for all $z \in \mathbf{C}^{n}$. Using (AU) we conclude that $f \in A_{\mathscr{K}}$. In view of the translation invariance of $A_{\mathscr{K}}$ the proof of the proposition is complete.

We shall now compare our notion of LAU-structure with Ehrenpreis' definition of PLAU-structures. For this definition we refer to Chapter IV.1 in [9]. The condition (b) stated there is essentially the same as condition (AU) above. More important is the fact that Ehrenpreis' localizability conditions (d) and (e) imply localizability in our sense.

Proposition 2. Let $\mathscr{K}$ be an $A U$-structure. If $\mathscr{K}$ is product localizable in the sense of Ehrenpreis then $\mathcal{K}$ is a $L A U$-structure.

Proof. Let $k \in \mathcal{K}$ be given. By (d) in [9, IV.1], there is a $k^{\prime} \in \mathcal{K}$ so that for any continuous $m \in \Re$ with $m(z) \leqslant k^{\prime}(z), z \in \mathbf{C}^{n}$, and every $z_{0} \in \mathbf{C}^{n}$ there is an entire 
function $f\left(\cdot ; z_{0}\right)$ such that $\left|f\left(z_{0} ; z_{0}\right)\right|>1$ and

$$
m\left(z_{0}\right)\left|f\left(z ; z_{0}\right)\right| \leqslant \min \left(k(z), m^{\prime}(z)\right), \quad z \in \mathbf{C}^{n},
$$

for some $m^{\prime} \in \mathscr{T}$ independent of $z_{0}$. Now choose a sequence $\left(z_{j}\right)_{1}^{\infty}, z_{j} \in \mathbf{C}^{n}$, such that $m(z) \leqslant \sup _{j} m\left(z_{j}\right)\left|f\left(z ; z_{j}\right)\right|, z \in \mathbf{C}^{n}$, and let $\varphi$ be the smallest upper semicontinuous majorant of the function $\sup _{j} \log \left(m\left(z_{j}\right)\left|f\left(\cdot ; z_{j}\right)\right|\right)$. Then $\varphi$ is plurisubharmonic, $e^{\varphi} \in \mathfrak{N}$ and $m(z) \leqslant e^{\varphi(z)}<k(z)$ for all $z \in \mathbf{C}^{n}$. Hence (L) is true for all continuous $m \in \mathfrak{T}$. As explained above (preceding Proposition 1) this suffices to conclude that $\mathscr{K}$ is localizable.

The product decomposability condition (i.e. the "P" in PLAU) which Ehrenpreis imposes on $\mathcal{K}$ is necessitated by the induction-on-dimension arguments occurring in the Oka-Cartan procedure. This condition is superfluous when working with Hörmander's results on cohomology with bounds instead.

3. The Fundamental Principle. The Fundamental Principle will be given for localizable analytically uniform spaces defined below. The definition of these spaces is essentially the same as Ehrenpreis' [9, Chapter IV.1].

Definition 2. Let $W$ be a locally convex space. Iff there exist a (L)AU-structure $\mathcal{K}$ and a separately continuous bilinear form $[\cdot, \cdot]$ on $W \times A_{\mathscr{K}}$ such that $W$ is isomorphic under this pairing (i.e. under map $w \rightarrow[w, \cdot], w \in W$ ) to the strong dual of $A_{\mathscr{K}}$ then $W$ will be called a (localizable) analytically uniform space (= (L)AU-space) with (L)AU-structure $\mathscr{K}$ and pairing $[\cdot, \cdot]$.

EXAMPLE. Let $\Omega \subset \mathbf{R}^{n}$ be open and convex. Then $\mathcal{E}(\Omega)$ is a LAU-space. The LAU-structure $\mathcal{K}$ is given in $\S 4$, example (iii), and the pairing $[\cdot, \cdot]$ between $\mathcal{E}(\Omega)$ and $A_{\mathscr{K}}$ is given by

$$
[u, f]=F^{-1} f(u), \quad u \in \mathcal{E}(\Omega) \text { and } f \in A_{\mathscr{K}} .
$$

Here $F$ denotes the Fourier-Laplace transform which maps $\mathcal{E}^{\prime}(\Omega)$, the space of distributions with compact support in $\Omega$, isomorphically onto $A_{\mathcal{K}}$ and is defined by $F \varphi(z)=\varphi\left(e^{-i<\cdot z\rangle}\right), z \in \mathbf{C}^{n}, \varphi \in \mathcal{E}^{\prime}(\Omega)$. Since $\mathcal{E}(\Omega)$ is a reflexive Fréchet space it is in fact a LAU-space in the sense of Definition 2.

Using the examples (iv) and (v) in $\$ 4$ we see in the same way that the Fréchet spaces $\mathcal{E}_{\omega}(\Omega)$ and $\mathcal{E}\left(\Omega,\left(M_{p}\right)\right)$ of ultra-differentiable functions in the sense of Beurling (with weight $\omega$ ) and of Roumieu (with weight sequence $\left(M_{p}\right)$ ), respectively, are LAU-spaces.

For the spaces $\mathcal{E}(\Omega), \mathcal{E}_{\omega}(\Omega)$ and $\mathcal{E}\left(\Omega,\left(M_{p}\right)\right)$ the following abstract definition of differential operators with constant coefficients on AU-spaces coincides with the usual definition if $D=-i \partial / \partial x$.

Definition 3. Let $W$ be an AU-space with AU-structure and pairing $[\cdot, \cdot]$. Let $P$ be an $L \times K$-matrix of polynomials. Denote by $P(D)$ the linear mapping from $W^{K}$ into $W^{L}$ which is the transpose with respect to $[\cdot, \cdot]$ of the linear mapping from $A_{\mathscr{K}}^{L}$ into $A_{\mathscr{K}}^{K}$ given by the matrix multiplication $f \rightarrow{ }^{t} P(-\cdot) f, f \in A_{\mathscr{K}}^{L}$. These operators $P(D)$ will be called differential operators with constant coefficients with symbol $P$.

With the definition (3.1) of the pairing for $\mathcal{E}(\Omega)$ we have

$$
u(x)=\delta_{x}(u)=\left[u, e^{-i\langle x, \cdot\rangle}\right]
$$


for all $u \in \mathcal{E}(\Omega)$ and $x \in \Omega\left(\delta_{x}=\right.$ Dirac measure at $\left.x\right)$. Therefore the integral representation (1.1) follows from the

Fundamental Principle. Let $W$ be a LAU-space with LAU-structure $\mathcal{K}$ and pairing $[\cdot, \cdot]$. Let $P(D): W^{K} \rightarrow W^{L}$ be a differential operator with constant coefficients and with symbol $P$. Then there exist (only depending on $P$ ) a positive integer $J$, a $J \times K$-matrix $\left(d_{j k}\left(z, \partial_{z}\right)\right)$ of differential operators with polynomial coefficients and algebraic varieties $V_{j} \subset\left\{z \in \mathbf{C}^{n}\right.$; rank $\left.{ }^{t} P(z)<K\right\}, j=1, \ldots, J$, such that $u=$ $\left(u_{1}, \ldots, u_{K}\right) \in W^{K}$ is a solution of the homogeneous equation $P(D) u=0$ if and only if for every $f \in A_{\mathscr{K}}$ and every $k=1, \ldots, K$

$$
\left[u_{k}, f\right]=\sum_{1}^{J} \int_{V_{j}} d_{j k}\left(z, \partial_{z}\right) f(-z) d \mu_{j}(z) .
$$

Here the $d \mu_{j}$ are Radon measures on $V_{j}$ depending on $u$ but not on $f$ which satisfy for some $k \in \mathcal{K}$

$$
\int_{V_{j}} k(z)\left|d \mu_{j}\right|(z)<+\infty, \quad j=1, \ldots, J .
$$

In particular, the integrals representing $u$ converge absolutely in $W$.

The hardest part in the proof of the Fundamental Principle is the proof of some deep theorems on division and extension of holomorphic functions. We shall only state them here. These results have been proved by Ehrenpreis [9] and Palamodov [16]. More recent proofs making heavy use of Hörmander's results on cohomology with bounds [12] have been given by Björk [6] and by Liess [14]. Björk's statement of these results is closest to the one which we give now.

Division- AND EXTENSION-ThEOREM. Let $Q$ be a $K \times L$-matrix of polynomials. Then there exist a positive integer $J, K$-vectors $d_{j}\left(z, \partial_{z}\right)$ of differential operators with polynomial coefficients and algebraic varieties $V_{j} \subset\left\{z \in \mathbf{C}^{n} ; \operatorname{rank} Q(z)<K\right\}, j=$ $1, \ldots, J$, and there is a positive constant $M$ such that the statements (Div) and (Ext) hold for every plurisubharmonic function $\varphi$ on $\mathbf{C}^{\boldsymbol{n}}$.

(Div) For every entire function $v: \mathbf{C}^{n} \rightarrow \mathrm{C}^{L}$ we have

$$
d_{j}\left(z, \partial_{z}\right)(Q v)(z)=0 \text { if } z \in V_{j}, j=1, \ldots, J .
$$

Conversely, if an entire function $g: \mathbf{C}^{n} \rightarrow \mathbf{C}^{K}$ satisfies $d_{j}\left(z, \partial_{z}\right) g(z)=0$ if $z \in V_{j}$, $j=1, \ldots, J$, then there exists an entire function $v: \mathbf{C}^{n} \rightarrow \mathbf{C}^{L}$ with $g=Q v$ in $\mathbf{C}^{n}$,

$$
\sup _{z \in \mathbf{C}^{n}}|v(z)| e^{-\varphi_{M}(z)}\left(2+|z|^{2}\right)^{-M}<\sup _{z \in \mathbf{C}^{n}}|g(z)| e^{-\varphi(z)} .
$$

Here and in the following $\varphi_{M}$ denotes the function

$$
\varphi_{M}(z)=\sup \left\{\varphi\left(z+z^{\prime}\right) ; z^{\prime} \in \mathbf{C}^{n},\left|z^{\prime}\right|<M\right\}, \quad z \in \mathbf{C}^{n} .
$$

This supremum is in fact a maximum since $\varphi$ is upper semicontinuous.

(Ext) For any entire function $g: \mathbf{C}^{n} \rightarrow \mathbf{C}^{K}$ there is another entire function $f$ : $\mathbf{C}^{n} \rightarrow \mathbf{C}^{K}$ such that

$$
\begin{gathered}
d_{j}\left(z, \partial_{z}\right)(f-g)(z)=0 \quad \text { if } z \in V_{j} \text { and } j=1, \ldots, J \\
\sup _{z \in \mathbf{C}^{n}}|f(z)| e^{-\varphi_{M}(z)}\left(2+|z|^{2}\right)^{-M}<\max _{j} \sup _{z \in V_{j}}\left|d_{j}\left(z, \partial_{z}\right) g(z)\right| e^{-\varphi(z)} .
\end{gathered}
$$


Since we cannot refer to the literature for exactly this statement we have to make some comments about it. One can prove this theorem by constructing $v$ and $f$ in (Div) and (Ext), respectively, at first locally near every point in $\mathbf{C}^{n}$ and then globally by correcting the resulting cochains with the help of Hörmander's results on cohomology with bounds. The local construction can be done keeping the necessary uniform bounds on the cochains if we use coverings of $\mathbf{C}^{n}$ with cubes of size $O\left(|\zeta|^{-m}\right)$ as the centers $\zeta$ of the cubes tend to infinity. Here $m$ is a constant depending only on $Q$. This is in fact the Semilocal Quotient Structure Theorem of Ehrenpreis. To apply Hörmander's technique (see [12, Chapter 7.6]) for passing from local to global we have to note two simple refinements to his approach. Firstly, the coverings to be used to define the cochain groups have to be chosen such that the sizes of the cubes belonging to the covering are bounded in a manner we have just indicated. Hörmander works with coverings consisting of congruent cubes. But it is easily checked that his results are not essentially changed when working with the more general coverings which are necessitated by the local construction. Secondly, we do not require the plurisubharmonic functions $\varphi$ to satisfy a Lipschitz-like condition $\left|\varphi(z)-\varphi\left(z^{\prime}\right)\right|<c$ if $\left|z-z^{\prime}\right|<1$ (see Theorem 7.6.10 in [12]). Hörmander uses this condition only to pass from $L^{2}$-estimates for holomorphic functions to corresponding sup-estimates. This is achieved with the inequality

$$
|u(z)| \leqslant C_{n} e^{\varphi_{1}(z)}\left(\int_{\left|z-z^{\prime}\right|<1}\left|u\left(z^{\prime}\right)\right|^{2} e^{-2 \varphi\left(z^{\prime}\right)} d \lambda\left(z^{\prime}\right)\right)^{1 / 2}
$$

which holds with a constant $C_{n}$ depending only on the dimension $n$ for every entire function $u$, every plurisubharmonic function $\varphi$ and all $z \in \mathbf{C}^{\boldsymbol{n}}$. Since this passage has to be made several times we have to know that the function $\varphi_{1}$ is plurisubharmonic if $\varphi$ is. Because of the uniformity in all plurisubharmonic functions (!) of the $L^{2}$-estimates for the $\bar{\partial}$-operator (see in particular Theorem 4.4.2 in [12]) we can then see that the constant $M>0$ occurring in (Div) and (Ext) may actually be chosen independent of $\varphi$.

Lemma 1. Let $\varphi$ be plurisubharmonic and let $M>0$. Then $\varphi_{M}$ (defined in (3.3)) is also plurisubharmonic.

Proof. Let $z \in \mathbf{C}^{n}$ and $c \in \mathbf{R}$ with $\varphi_{M}(z)<c$ be given. By the upper semicontinuity of $\varphi$ there exists for every $z^{\prime} \in \mathrm{C}^{n}$ with $\left|z^{\prime}\right|<M$ a positive $\varepsilon$ such that $\varphi\left(\zeta+\zeta^{\prime}\right)<c$ for all $\zeta, \zeta^{\prime} \in \mathbf{C}^{n}$ with $|z-\zeta|<\varepsilon$ and $\left|z^{\prime}-\zeta^{\prime}\right|<\varepsilon$. From the Heine-Borel theorem we therefore get $\varphi_{M}(\zeta)<c$ if $|z-\zeta|<\delta$ for some positive $\delta$. Hence $\varphi_{M}$ is upper semicontinuous and therefore also plurisubharmonic by Theorem 1.6.2 in [12].

Let us now show that

(Div) and (Ext) imply the Fundamental Principle. A "function" $u \in W^{K}$ is a solution of the homogeneous equation $P(D) u=0$ if and only if it defines via the pairing $[\cdot, \cdot]$ a continuous linear functional on the quotient space

$$
A_{\mathscr{K}}^{K} / \overline{Q A_{\mathscr{K}}^{L}}
$$


where $Q$ is the $K \times L$-matrix of polynomials given by $Q(z)={ }^{t} P(-z), z \in \mathbf{C}^{n}$. This follows by duality from the definition of differential operators on LAU-spaces.

Choose $J, d_{j}$ and $V_{j}$ as in the Division- and Extension-Theorem. Let $\tilde{C}_{\mathscr{K}}$ denote the locally convex space consisting of all $J$-tuples $\left(h_{1}, \ldots, h_{j}\right)$ of continuous $\mathbf{C}^{K}$-valued functions $h_{j}$ on $V_{j}$ with $\left|h_{j}(z)\right| / k(z) \rightarrow 0$ as $z \rightarrow \infty, z \in V_{j}, j=1, \ldots, J$, for every $k \in \mathcal{K}$ and carrying the topology defined by the family (parametrized by $k \in \mathscr{K}$ ) of seminorms

$$
\left(h_{1}, \ldots, h_{j}\right) \rightarrow \max _{j} \sup _{z \in V_{j}}\left|h_{j}(z)\right| / k(z) .
$$

Let $N$ denote the linear mapping from $A_{\mathscr{K}}^{K}$ into $\tilde{C}_{\mathscr{K}}$ given by $f \rightarrow$ $\left(\left.d_{1}\left(\cdot, \partial_{z}\right) f\right|_{V_{1}}, \ldots,\left.d_{J}\left(\cdot, \partial_{z}\right) f\right|_{V_{J}}\right)$.

Since $\mathscr{K}$ is an AU-structure, $N$ is well defined and continuous. We shall show that the kernel of $N$ coincides with $\overline{Q A_{\mathscr{K}}^{L}}$ and that $N$ is a homomorphism (in the sense of functional analysis). From this would follow that $N$ identifies the quotient space (3.4) with a subspace of $\tilde{C}_{\mathscr{T}}$ and the proof of the Fundamental Principle is then completed by a standard application of the Hahn-Banach theorem and the Riesz representation theorem (as in the proof of Proposition 1).

Since $N$ is continuous it follows from the first part of (Div) that $\overline{Q A_{\mathscr{K}}^{L}}$ is contained in the kernel of $N$. Now let $g \in A_{\mathscr{K}}^{K}$ be given with $N g=0$. Since $\mathscr{K}$ is localizable (i.e. (L) holds) there exists a plurisubharmonic function $\varphi$ with $e^{\varphi} \in$ $\mathscr{R}(\mathcal{K})$ and $|g(z)| \leqslant e^{\varphi(z)}, z \in \mathbf{C}^{n}$. Therefore there exists by (Div) an entire function $v: \mathbf{C}^{n} \rightarrow \mathbf{C}^{L}$ with $g(z)=Q(z) v(z), z \in \mathbf{C}^{n}$, and (3.2). Applying (AU) repeatedly we see that $v \in A_{\mathscr{K}}^{L}$. So $Q A_{\mathscr{K}}^{L}$ coincides with $\operatorname{ker} N$ and is in particular closed.

Now let us prove that $N$ is a homomorphism. Let $k_{0} \in \mathscr{K}$ be given. With $M>0$ as in (Ext) choose $k \in \mathscr{K}$ with

$$
k\left(z+z^{\prime}\right)\left(2+|z|^{2}\right)^{M}<k_{0}(z),
$$

for all $z, z^{\prime} \in \mathbf{C}^{n}$ with $\left|z^{\prime}\right|<M$. This is possible by (AU). Choose $k^{\prime} \in \mathscr{K}$ (depending on $k$ ) as in (L). Let $g \in A_{\mathscr{K}}^{K}$ with $\left|d_{j}\left(z, \partial_{z}\right) g(z)\right|<k^{\prime}(z)$ if $z \in V_{j}, j=1, \ldots, J$, be given. We are done once we have exhibited an entire function $f \in A_{\mathscr{K}}^{K}$ with $N f=N g$ and $|f(z)|<k_{0}(z), z \in \mathbf{C}^{n}$. By the localizability (L) of $\mathscr{K}$ there exists a plurisubharmonic function $\varphi$ (depending on $g$ ) with $e^{\varphi} \in \mathscr{N}(\mathscr{K}),\left|d_{j}\left(z, \partial_{z}\right) g(z)\right|<$ $e^{\varphi(z)}$ if $z \in V_{j}, j=1, \ldots, J$, and

$$
e^{\varphi(z)}<k(z), \quad z \in \mathbf{C}^{n} .
$$

From (Ext) now follows the existence of an entire function $f: \mathbf{C}^{n} \rightarrow \mathbf{C}^{K}$ with $N(f-g)=0,|f(z)|<\left(2+|z|^{2}\right)^{M} e^{\varphi_{M}(z)}, z \in \mathbf{C}^{n}$. Using (AU) we get $f \in A_{\mathscr{K}}^{K}$ and with (3.5) and (3.6) we obtain $|f(z)| \leqslant k_{0}(z)$ for every $z \in \mathbf{C}^{n}$, as desired. This completes the proof.

4. Some examples of LAU-structures. Many spaces of entire functions occurring in Fourier analysis are naturally given as locally convex inductive limit spaces defined by weight functions. By the Paley-Wiener theorem the space of Fourier-Laplace transforms of $\mathcal{E}^{\prime}(\Omega)$, the space of distributions with compact 
support in $\Omega, \Omega \subset \mathbf{R}^{n}$ open and convex, is an example for such a space. Our purpose is to show that a rather large and important class of inductive limit spaces of entire functions (containing in particular the example just mentioned) can be given LAU-structures defining the spaces algebraically and topologically.

Let $\Sigma=\left(\sigma_{j}\right)_{1}^{\infty}$ be a sequence of continuous real valued functions $\sigma_{j}$ on $\mathbf{C}^{n}$ such that for all $j$

$$
\sigma_{j}(z) \leqslant \sigma_{j+1}(z), \quad z \in \mathbf{C}^{n},
$$

and

$$
\sigma_{j}(z)-\sigma_{j+1}(z) \rightarrow-\infty \text { as } z \rightarrow \infty .
$$

For complex valued functions $f$ or $\mathbf{C}^{n}$ set

$$
q_{j}(f)=\sup _{z \in \mathbf{C}^{n}}|f(z)| e^{-\sigma_{j}(z)}, \quad j=1,2, \ldots
$$

Let $A[\Sigma]$ be the space of all entire functions $f$ which satisfy $q_{j}(f)<+\infty$ for some $j$ depending on $f$. Equip $A[\Sigma]$ with the natural locally convex inductive limit topology. Analogously, let $C[\Sigma]$ be the locally convex inductive limit space consisting of all continuous complex valued functions $f$ on $\mathbf{C}^{n}$ with $q_{j}(f)<+\infty$ for some $j=j(f)$. Let $\mathscr{K}=\mathscr{K}_{\Sigma}$ be the set of all continuous functions $k$ on $\mathbf{C}^{n}$ which are given with some sequence $\left(\delta_{j}\right)_{1}^{\infty}$ of positive numbers $\delta_{j}$ by

$$
k(z)=\sup _{j} \delta_{j} e^{\sigma_{j}(z)} \quad \text { for all } z \in \mathbf{C}^{n} .
$$

Recall that a (LS)-space is a countable inductive limit of Banach spaces with compact spectral mappings. It is well known (see e.g. [10, §25]) that a (LS)-space is Hausdorff if the spectral mappings are injective and that Hausdorff (LS)-spaces are in particular Montel spaces.

THEOREM 1. Let $\Sigma, A[\Sigma]$ and $\mathscr{K}=\mathcal{K}_{\Sigma}$ be as above. Then $A[\Sigma]$ is a (LS)-space and $A[\Sigma]=A_{\mathscr{K}}$ as locally convex spaces. If for every positive integer $j$ there is another positive integer $l$ such that $\sigma_{j}\left(z+z^{\prime}\right)+\log \left(2+|z|^{2}\right)-\sigma_{l}(z)<C$ for all $z, z^{\prime} \in \mathbf{C}^{n}$ with $\left|z^{\prime}\right|<1$ and some finite constant $C$ independent of $z$ and $z^{\prime}$, then $\mathcal{K}$ is an $A U$-structure. If, in addition, there exists a sequence of plurisubharmonic functions $\left(\varphi_{j}\right)_{1}^{\infty}$ with

$$
\sigma_{j}(z) \leqslant \varphi_{j}(z) \leqslant \sigma_{j+1}(z), \quad z \in \mathbf{C}^{n},
$$

for all $j=1,2, \ldots$, then $\mathscr{K}$ is a $L A U$-structure.

REMARK. Except for the localizability statement, this theorem has been proved by Taylor [17]. He uses Hörmander's results on $\bar{\partial}$-solvability in his proof and therefore assumes the $\sigma_{j}$ to be plurisubharmonic. A purely functional-analytic proof of Taylor's theorem has been given by Bierstedt, Meise and Summers (see [3] and [4]). We shall essentially follow their method in the

Proof of Theorem 1. From Montel's theorem and (4.2) it follows easily that every sequence of entire functions which is bounded with respect to the seminorm $q_{j}$ has a subsequence which converges with respect to the seminorm $q_{j+1}$. This implies that $A[\Sigma]$ is a (LS)-space. 
To show $A[\Sigma]=A_{\mathscr{K}}$ let us first prove the corresponding result $C[\Sigma]=C_{\mathscr{K}}$ for the associated spaces of continuous functions. Provided $\mathscr{K}$ is nonempty, $C[\Sigma]$ is obviously continuously imbedded in $C_{\mathscr{K}}$. To prove the converse let $U$ be a closed absolutely convex neighbourhood of zero in $C[\Sigma]$. We have to find $k \in \mathcal{K}$ such that $\left\{f \in C[\Sigma] ; p_{k}(f)<1\right\} \subset U$. By the definition of the inductive limit $C[\Sigma]$ there exists a sequence $\left(\varepsilon_{j}\right)_{1}^{\infty}$ of positive numbers such that

$$
\bigcup_{j=1}^{\infty}\left\{f \in C[\Sigma] ; q_{j}(f)<\varepsilon_{j}\right\} \subset U .
$$

Choose a locally finite partition of unity $\left(\alpha_{l}\right)_{1}^{\infty}$ on $\mathbf{C}^{n}, \alpha_{l}$ continuous with compact support, such that

$$
\exp \left(\sigma_{l-1}(z)-\sigma_{l}(z)\right)<2^{-l} \varepsilon_{l} \quad \text { if } \alpha_{l}(z) \neq 0 \text { and } l>1 .
$$

Choose a sequence $\left(\delta_{j}\right)_{1}^{\infty}$ of positive numbers $\delta_{j}<1$ such that

$$
\delta_{j} \exp \left(\sigma_{j}(z)-\sigma_{l}(z)\right)<\min \left(\delta_{l}, 2^{-l} \varepsilon_{l}\right) \quad \text { if } \alpha_{l}(z) \neq 0,1<l<j .
$$

Since this involves for every $\delta_{j}$ really only finitely many conditions such a sequence $\left(\delta_{j}\right)_{1}^{\infty}$ can be found. Now set

$$
k(z)=\sup _{j=1,2, \ldots} \delta_{j} e^{\sigma_{j}(z)}, \quad z \in \mathbf{C}^{n} .
$$

It follows from (4.6) that this supremum is locally a maximum and therefore $k$ is continuous and $k \in \mathscr{K}$. In particular, $\mathscr{K}$ is nonempty. To show that $p_{k}$ is the required seminorm let $f \in C[\Sigma]$ with

$$
|f(z)|<k(z), \quad z \in \mathbf{C}^{n},
$$

given. Defining $\sigma_{0}=\sigma_{1}$ and recalling that $\delta_{j}<1$ we then have by (4.1) and (4.7)

$$
\begin{aligned}
q_{l}\left(\alpha_{l} f\right) & \leqslant \sup _{\alpha_{l}(z) \neq 0} k(z) \exp \left(-\sigma_{l}(z)\right) \\
& <\sup _{\alpha_{l}(z) \neq 0} \max \left(\exp \left(\sigma_{l-1}(z)-\sigma_{l}(z)\right), \sup _{l<j} \delta_{j} \exp \left(\sigma_{j}(z)-\sigma_{l}(z)\right)\right) .
\end{aligned}
$$

From (4.5) and (4.6) now follows that $q_{l}\left(\alpha_{l} f\right)<2^{-l} \varepsilon_{l}$ for all $l=1,2, \ldots$

From (4.4) and the assumptions on $U$ we therefore obtain $f=\Sigma_{1}^{\infty} 2^{-l}\left(2^{l} \alpha_{l} f\right) \in U$ provided this series converges with respect to the inductive limit topology. As for this, first note that $q_{j}(f)<+\infty$ for some $j$. Since $\exp \left(\sigma_{j}(z)-\sigma_{j+1}(z)\right)$ tends to zero with $1 /|z|$ (see (4.2)) it is easily seen that the above series converges with respect to the seminorm $q_{j+1}$ and hence converges in the inductive limit topology. Thus we have now shown that the subspace topology on $C[\Sigma]$ inherited from $C_{\mathscr{K}}$ coincides with its original inductive limit topology. To show that $C_{\mathscr{K}} \subset C[\Sigma]$ we prove that more generally any bounded subset of $C_{\mathscr{X}}$ is already bounded with respect to some seminorm $q_{j}$. Assume that, on the contrary, there exists a bounded subset $B$ of $C_{\mathscr{K}}$ which is unbounded with respect to every seminorm $q_{l}$. We may thus choose a sequence $\left(f_{l}\right)_{1}^{\infty}$ in $B$ with

$$
\left|f_{l}\left(z_{l}\right)\right|>l \cdot e^{\sigma_{l}\left(z_{l}\right)}, \quad l=1,2, \ldots,
$$

for a sequence $\left(z_{l}\right)_{1}^{\infty}$ in $\mathbf{C}^{n}$ which tends to infinity. Choose a sequence $\left(\delta_{j}\right)_{1}^{\infty}$ of positive numbers $\delta_{j}<1$ such that $\delta_{j} e^{\sigma_{j}\left(z_{l}\right)}<e^{\sigma_{l}\left(z_{l}\right)}, 1<l<j$, and such that the 
function $k(z)=\sup _{j} \delta_{j} e^{\sigma_{j}(z)}, z \in \mathbf{C}^{n}$, is continuous and therefore in $\mathcal{K}$. Then we have $k\left(z_{l}\right) \leqslant e^{\sigma_{l}\left(z_{l}\right)}$ for all $l$. But this implies together with (4.8) that the sequence $\left(f_{l}\right)_{1}^{\infty}$ cannot be bounded with respect to the seminorm $p_{k}$. This contradicts the assumption that $B$ is bounded in $C_{\mathscr{K}}$. So we have shown that $C_{\mathscr{K}}=C[\Sigma]$ and that this space has a fundamental sequence of bounded subsets. This implies that $C[\Sigma]$ is a (DF)-space (see e.g. [13, §29]).

These facts enable us to apply a homomorphism lemma of A. Baernstein II to the inclusion mapping $A[\Sigma] \hookrightarrow C[\Sigma]$ to derive the (nontrivial) fact that $A[\Sigma]$ in its inductive limit topology is actually a subspace of $C[\Sigma]$. In view of $C[\Sigma]=C_{\mathscr{C}}$ we then know that $A[\Sigma]=A_{\mathscr{K}}$ as locally convex spaces.

Homomorphism Lemma (BAERNSTEIN). Let $E$ be a Montel space and let $F$ be $a$ (DF)-space. Let $T$ be a continuous linear mapping from $E$ into $F$ with the property that $T^{-1}(B)$ is bounded whenever $B$ is. Then $T^{-1}$ is a continuous linear mapping from $T E$ onto $E$.

For a proof see $\$ 2$ in [1].

Now assume that there is a sequence $(l(j))_{j=1}^{\infty}$ of positive integers $l(j)$ and a sequence $\left(a_{j}\right)_{1}^{\infty}$ of positive reals $a_{j}$ such that for all $j$

$$
\sigma_{j}\left(z+z^{\prime}\right)+\log \left(2+|z|^{2}\right)<\sigma_{l(j)}(z)-\log a_{j}
$$

if $z, z^{\prime} \in \mathbf{C}^{n}$ with $\left|z^{\prime}\right|<1$. Let $k \in \mathscr{K}$ be given. More specifically, let us assume that $k(z)=\sup _{j} \delta_{j} e^{\sigma_{j}(z)}, z \in \mathbf{C}^{n}$, for a sequence $\left(\delta_{j}\right)_{1}^{\infty}$ of positive numbers. Making constructions as above we can find $k^{\prime} \in \mathscr{K}$ with

$$
k^{\prime}(z) \leqslant \sup _{j} a_{j} \cdot \delta_{l(j)} e^{\sigma_{j}(z)}, \quad z \in \mathbf{C}^{n}
$$

From (4.9) now follows

$$
k^{\prime}\left(z+z^{\prime}\right)\left(2+|z|^{2}\right)<k(z) \text { if } z, z^{\prime} \in \mathbf{C}^{n},\left|z^{\prime}\right|<1 .
$$

Thus $\mathscr{K}$ is an AU-structure.

Now assume that, in addition, there exists a sequence of plurisubharmonic functions $\left(\varphi_{j}\right)_{1}^{\infty}$ with (4.3). Let $k \in \mathcal{K}$ be given. Choose a sequence $\left(\varepsilon_{j}\right)_{1}^{\infty}$ of positive reals with

$$
\varepsilon_{l} e^{\varphi_{l}(z)}<\max _{j<l} \varepsilon_{j} e^{\varphi_{j}(z)} \quad \text { if } z \in \mathbf{C}^{n},|z|<l,
$$

for all $l=1,2, \ldots$, and choose $k^{\prime} \in \mathcal{K}$ with

$$
k^{\prime}(z)<\sup _{j} \varepsilon_{j} e^{\varphi_{j}(z)}<k(z), \quad z \in \mathbf{C}^{n} .
$$

Using (4.3) and the continuity of $\sigma_{j}$ such choices can be made. Let $m \in \mathscr{T}(\mathcal{K})$ be continuous with

$$
m(z)<k^{\prime}(z), \quad z \in \mathbf{C}^{n} .
$$

Since $\{m\}$ is bounded in $C_{\mathscr{K}}$ we already know that $m(z)<l \cdot e^{\varphi_{1}(z)}, z \in \mathbf{C}^{n}$, for some $l \in \mathbf{N}$. By (4.2) we can find an integer $l_{0}>l+2$ with

$$
m(z)<\varepsilon_{l+2} e^{\varphi_{1+2}(z)} \quad \text { if } z \in \mathbf{C}^{n},|z|>l_{0} .
$$


By (4.10) and (4.11) we have

$$
k^{\prime}(z)<\max _{j<l_{0}} \varepsilon_{j} e^{\varphi_{j}(z)} \quad \text { if } z \in \mathbf{C}^{n},|z|<l_{0} .
$$

This results with (4.11), (4.12) and (4.13) in $m(z)<e^{\varphi(z)}<k(z), z \in \mathbf{C}^{n}$, where $\varphi(z)=\max _{j<l_{0}}\left(\varphi_{j}(z)+\log \varepsilon_{j}\right), z \in \mathbf{C}^{n}$.

Since obviously $e^{\varphi} \in \mathscr{T}(\mathscr{K})$ and since $\varphi$ is plurisubharmonic we have shown that $\mathcal{K}$ is localizable (recall that the continuity assumption on $m$ is no restriction when dealing with AU-structures). This completes the proof of the theorem.

ExAMPLES. Theorem 1 applies to the following examples of spaces $A[\Sigma], \Sigma=$ $\left(\sigma_{j}\right)_{1}^{\infty}$, in full strength to give localizable analytically uniform structures $\mathscr{K}=\mathscr{K}_{\Sigma}$ with $A[\Sigma]=A_{\mathscr{K}}$.

(i) The space of polynomials in $n$ variables with complex coefficients. In fact, this is the space $A[\Sigma]$ with the sequence $\Sigma=\left(\sigma_{j}\right)_{1}^{\infty}$ consisting of the plurisubharmonic functions

$$
\sigma_{j}(z)=j \cdot \log \left(2+|z|^{2}\right), \quad z \in \mathbf{C}^{n}, j=1,2, \ldots
$$

For this example the proof of the Fundamental Principle leads to a generalization of Hilbert's Nullstellensatz to submodules $M$ of $\mathcal{P}^{K}(K \in \mathrm{N}, \mathcal{P}=$ ring of complex valued polynomials). This follows from the geometric description which one obtains for the quotient module $\mathcal{P}^{K} / M$. This example was given by Ehrenpreis (see [9, Chapter V.8]).

(ii) The space of Laplace transforms of analytic functionals on an open convex subset $\Omega \subset C^{n}$. By the Ehrenpreis-Martineau theorem (see [9, Theorem 5.21] or [12, Theorem 4.5.3]) this is the locally convex space $A[\Sigma], \Sigma=\left(\sigma_{j}\right)_{1}^{\infty}$, with

$$
\sigma_{j}(z)=\sup _{\zeta \in K_{j}} \operatorname{Re}\langle z, \zeta\rangle, \quad j=1,2, \ldots,
$$

for a sequence $\left(K_{j}\right)_{1}^{\infty}$ of compact convex $K_{j} \subset \Omega$ exhausting $\Omega$ and satisfying $K_{j} \subset$ int $K_{j+1}$. In the case $\Omega=\mathbf{C}^{n}$ this is the space of all entire functions of exponential growth. Being continuous and the supremum of plurisubharmonic functions the functions $\sigma_{j}$ are seen to be plurisubharmonic. This allows us to regard the space $\mathcal{H}(\Omega)$ of holomorphic functions in an open convex subset $\Omega$ of $\mathbf{C}^{\boldsymbol{n}}$ in a natural way as a LAU-space. This example was for $\Omega=\mathbf{C}^{\mathbf{n}}$ considered in detail by Ehrenpreis (see [9, Chapter V.1]). He proved $\mathcal{H}\left(\mathbf{C}^{n}\right)$ to be a LAU-space.

(iii) The space of Fourier-Laplace transforms of the space $\mathcal{E}^{\prime}(\Omega)$ of distributions with compact support in a convex open subset $\Omega$ of $\mathbf{R}^{n}$. In fact, by the Paley-WienerSchwartz theorem the Fourier-Laplace transform maps $\mathcal{E}^{\prime}(\Omega)$ isomorphically onto $A[\Sigma]$ if we let $\Sigma=\left(\sigma_{j}\right)_{j \in N}$ consist of the functions

$$
\sigma_{j}(z)=j \cdot \log \left(2+|z|^{2}\right)+H_{j}(\operatorname{Im} z), \quad z \in \mathbf{C}^{n}, j \in \mathbf{N} .
$$

Here $\left(H_{j}\right)_{j \in N}$ is the sequence of supporting functions corresponding to a fixed sequence $\left(K_{j}\right)_{j \in \mathrm{N}}$ of compact convex subsets $K_{j} \subset \Omega$ with $K_{j} \subset$ int $K_{j+1}$ which exhausts $\Omega$. This means that $H_{j}(\eta)=\sup \left\{\langle x, \eta\rangle ; x \in K_{j}\right\}, \eta \in \mathbf{R}^{n}$. The functions $H_{j}$ are plurisubharmonic since they are continuous and a supremum of plurisubharmonic functions. Therefore all functions $\sigma_{j}$ are plurisubharmonic. 
This example was for polyhedra $\Omega \subset \mathbf{R}^{n}$ shown to be LAU by Ehrenpreis (see [9, Chapters V.5 and IV.5]). The statement of analytical uniformity follows for arbitrary open convex $\Omega$ readily from Taylor's theorem [17].

(iv) The space of Fourier-Laplace transforms of the space of Beurling ultradistributions $\mathcal{E}_{\omega}^{\prime}(\Omega)$ with compact support in an open convex subset $\Omega$ of $\mathbf{R}^{n}$. For the definition of these ultradistributions and their basic properties we refer to [5]. Here $\omega$ is assumed to be a continuous real valued function on $\mathbf{R}^{n}$ satisfying the conditions

$(\alpha) 0<\omega(0) \leqslant \omega(\xi+\eta) \leqslant \omega(\xi)+\omega(\eta)$ for all $\xi, \eta \in \mathbf{R}^{n}$,

( $\beta) \int \omega(\xi)(1+|\xi|)^{-n-1} d \xi<+\infty$, and

$(\gamma) \omega(\xi) \geqslant a+b \cdot \log (1+|\xi|), \xi \in \mathbf{R}^{n}$, for some real $a$ and positive $b$.

The preceding example (iii) is contained in example (iv) since the Schwartz distributions correspond to $\omega(\xi)=\log (1+|\xi|)$.

By the Paley-Wiener theorem for Beurling ultradistributions (see Theorem 1.8.14 in [5]) the Fourier-Laplace transform maps $\mathcal{E}_{\omega}^{\prime}(\Omega)$ isomorphically onto $A[\Sigma]$ if we let $\Sigma=\left(\sigma_{j}\right)_{j \in \mathrm{N}}$ consist of the functions $\sigma_{j}(z)=j+j \cdot \omega(\operatorname{Re} z)+H_{j}(\operatorname{Im} z), z \in \mathbf{C}^{n}$, $j \in \mathbf{N}$. Here $\left(H_{j}\right)_{j \in \mathbf{N}}$ is chosen as in example (iii).

It is immediately clear that Theorem 1 applies to give $A[\Sigma]=A_{\mathscr{K}}$ for a certain AU-structure. But the localizability is not obvious because $\omega(\operatorname{Re} \cdot)$ need not be plurisubharmonic. However, since there is for every $j \in \mathbf{N}$ a positive $\varepsilon_{j}$ with $H_{j}(\eta)+\varepsilon_{j}|\eta| \leqslant H_{j+1}(\eta), \eta \in \mathbf{R}^{n}$, we can infer the localizability of $\mathscr{K}$ from statement (a) in

LEMMA 2. Let $\omega$ be a continuous real valued function on $\mathbf{R}^{n}$ with $(\alpha),(\beta)$ and $(\gamma)$. Then there exist for every $\varepsilon>0$ plurisubharmonic functions $\varphi_{+}$and $\varphi_{-}$such that

(a) $-c-\varepsilon|\operatorname{Im} z|<\varphi_{+}(z)-\omega(\operatorname{Re} z)<\varepsilon|\operatorname{Im} z|$,

(b) $-c-\varepsilon|\operatorname{Im} z|<\varphi_{-}(z)+\omega(\operatorname{Re} z)<\varepsilon|\operatorname{Im} z|$,

for all $z \in \mathbf{C}^{n}$ with a constant $c$ independent of $z$.

REMARK. More generally, one can approximate Lipschitz functions by plurisubharmonic functions (see [15, Proposition 2.1]). However, the approximation for large $|\operatorname{Im} z|$ given in [15] is too crude for our purposes.

Proof of Lemma 2. Let $\varepsilon>0$. Choose an entire function $f$ with $f(0) \neq 0$ and

$$
|f(z)|<\exp (-\omega(-\operatorname{Re} z)+\varepsilon|\operatorname{Im} z|), \quad z \in \mathbf{C}^{n} .
$$

The existence of such a function $f$ is related to the existence of nontrivial test functions in $\mathscr{D}_{\omega}$ with support contained in $\left\{x \in \mathbf{R}^{n} ;|x|<\varepsilon\right\}$ (see [5]). In fact, $f$ can be chosen as the Fourier-Laplace transform of such a test function. Now choose $\delta>0$ with $|f(z)| \geqslant \delta$ if $|z|<\delta$. Choose a sequence $\left(z_{j}\right)_{1}^{\infty}, z_{j} \in \mathbf{C}^{n}$, such that the union of all balls with center $z_{j}$ and radius $\delta$ covers $\mathbf{C}^{n}$. Let $\varphi_{+}$be the smallest upper semicontinuous majorant of the function

$$
z \rightarrow \sup _{j}\left(\log \left|f\left(z-z_{j}\right)\right|+\omega\left(\operatorname{Re} z_{j}\right)-\varepsilon\left|\operatorname{Im} z_{j}\right|\right) .
$$

Then $\varphi_{+}$is plurisubharmonic on $\mathbf{C}^{n}$. 
Given $z$ and $z_{j}$ we have, using ( $\left.\alpha\right)$ and (4.14),

$$
\log \left|f\left(z-z_{j}\right)\right| \leqslant-\omega\left(\operatorname{Re} z_{j}\right)+\omega(\operatorname{Re} z)+\varepsilon|\operatorname{Im} z|+\varepsilon\left|\operatorname{Im} z_{j}\right| \cdot
$$

From this the second inequality in (a) follows. Using ( $\alpha$ ) we obtain for $\left|z-z_{j}\right|<\delta$,

$$
\begin{aligned}
\log \left|f\left(z-z_{j}\right)\right|+ & \omega\left(\operatorname{Re} z_{j}\right)-\varepsilon\left|\operatorname{Im} z_{j}\right| \\
& \geqslant \log \delta-\omega\left(\operatorname{Re} z-\operatorname{Re} z_{j}\right)+\omega(\operatorname{Re} z)-\varepsilon|\operatorname{Im} z|-\varepsilon \delta .
\end{aligned}
$$

Hence the first inequality in (a) follows with $c=-\log \delta+\varepsilon \delta+\sup \{\omega(\xi) ;|\xi|<\delta\}$. Choosing $f$ with

$$
|f(z)| \leqslant \exp (-\omega(\operatorname{Re} z)+\varepsilon|\operatorname{Im} z|), \quad z \in \mathbf{C}^{\mathbf{n}},
$$

instead of (4.14) and defining $\varphi_{-}$as the smallest upper semicontinuous majorant of the function $z \rightarrow \sup _{j}\left(\log \left|f\left(z-z_{j}\right)\right|-\omega\left(\operatorname{Re} z_{j}\right)-\varepsilon\left|\operatorname{Im} z_{j}\right|\right)$ we can derive (b). The proof is analogous to the proof of (a) and is therefore left to the reader.

The space of Beurling ultradifferentiable functions on $\mathbf{R}^{n}$ was considered by Berenstein and Dostal (see [2, Chapter II, §2]). There it is shown that $\mathcal{E}_{\omega}$ is analytically uniform. The localizability of $\mathcal{E}_{\omega}$ is not shown there.

(v) The space of Fourier-Laplace transforms of the space of Roumieu ultradistributions $\mathcal{E}^{\prime}\left(\Omega,\left(M_{p}\right)\right)$ with compact support in an open convex subset $\Omega$ of $\mathbf{R}^{n}$. Here $\left(M_{p}\right)$, $p \in \mathbf{N}^{n}$, is a logarithmically convex sequence satisfying a nonquasianalyticity condition. For a precise definition we refer to [7] (in the notation of [7], $\mathcal{E}^{\prime}\left(\Omega,\left(M_{p}\right)\right.$ ) is the space $\mathcal{E}_{0}^{\prime}\left(M_{(p)}, \Omega\right)$ ). By the Paley-Wiener theorem for Roumieu ultradistributions (see e.g. [7, p. 37]) the Fourier-Laplace transform maps $\mathcal{E}^{\prime}\left(\Omega,\left(M_{p}\right)\right)$ isomorphically onto $A[\Sigma]$ if we let $\Sigma=\left(\sigma_{j}\right)_{j \in N}$ consist of the functions $\sigma_{j}(z)=M(j z)+$ $H_{j}(\operatorname{Im} z), z \in \mathbf{C}^{n}, j \in \mathbf{N}$. Here again $\left(H_{j}\right)_{j \in \mathbf{N}}$ is chosen as in example (iii) and

$$
M(z)=\sup _{p \in \mathrm{N}^{n}} \log \left(\left|z^{p}\right| / M_{p}\right), z \in \mathbf{C}^{n} .
$$

The nonquasianalyticity of $\left(M_{p}\right)$ implies that $M_{p}^{1 /|p|}$ tends to infinity with $|p|$. Hence the supremum defining $M$ is locally a maximum and so $M$ is continuous and plurisubharmonic. Therefore all functions $\sigma_{j}$ are continuous and plurisubharmonic and Theorem 1 applies to give $A[\Sigma]=A_{\mathscr{K}}$ for some LAU-structure $\mathcal{K}$.

Chou has shown (see [7, Chapter $I, \S 2.3])$ that $\mathcal{E}\left(\Omega,\left(M_{p}\right)\right)$ is analytically uniform. This and the localizability were also proved by Ehrenpreis (see [9, Chapter V.6]) in case $\Omega=\mathbf{R}^{n}$.

REMARK. The statements on analytical uniformity in the foregoing examples contain nothing new. Localizability, however, seems to be difficult to verify when using the methods of [9] for spaces like $\mathcal{E}(\Omega)$ when $\Omega \subset \mathbf{R}^{n}$ is open and convex but no polyhedron and a complete proof has, in fact, not been carried out in [9]. With the methods used here these examples can be handled rather easily, however. This is of course mainly due to our new definition of localizability (see condition (L)) which eases that of Ehrenpreis considerably.

REMARK. Ehrenpreis has shown that the space of Fourier-Laplace transforms of the space of Schwartz test functions $\mathscr{D}\left(\mathbf{R}^{n}\right)$ admits an analytically uniform structure and he thus proved that $\mathscr{D}^{\prime}\left(\mathbf{R}^{n}\right)$ is an AU-space (see [9, Chapter V.4]). This has been generalized by Berenstein and Dostal [2] to a proof that the space of Beurling 
ultradistributions $\mathscr{D}_{\omega}^{\prime}\left(\mathbf{R}^{n}\right)$ is an AU-space and by Chou [7] to a proof that the space of Roumieu ultradistributions $\mathscr{D}^{\prime}\left(\Omega,\left(M_{p}\right)\right)$ in an open convex subset $\Omega$ of $\mathbf{R}^{n}$ is an AU-space. In [9] and [2] also the question of localizability of $\mathscr{D}^{\prime}\left(\mathbf{R}^{n}\right)$ and $\mathscr{D}_{\omega}^{\prime}\left(\mathbf{R}^{n}\right)$, respectively, is studied. However, the definition of localizability used in [2] (see Definition 3(viii) on p. 21) only requires for every $m \in \mathfrak{N}(\mathcal{K})$ the existence of a plurisubharmonic function $\varphi$ with $m(z) \leqslant e^{\varphi(z)}, z \in \mathbf{C}^{n}$, and $e^{\varphi} \in \mathfrak{N}$. But when working with this definition the proof of the Fundamental Principle given in [2, Chapter IV, §2] is not complete. Note that our stronger localizability condition (L) is essential for proving $N$ to be a homomorphism (last part of §3). The point in all this is that one has to insure that a subset $\Re_{1} \subset \mathfrak{R}(\mathscr{K})$ is a sufficient BAU-structure in the sense of Ehrenpreis (see [9, p. 97]) when $\mathscr{N}(\mathscr{K})$ is to be replaced by $\Re_{1}$. However, even in [9] this point is not always treated carefully. On p. 127 in [9] it is implicitly assumed that all functions $B^{\prime} \exp \left(A^{\prime}|\cdot|\right)$ form a sufficient BAUstructure for the space of holomorphic functions of exponential growth. But there is no reason for this to be true. A sufficient BAU-structure is easily found though when looking at the last part of the proof of our Theoem 1. Unfortunately, we have not yet fully understood the localizability proof for $\mathscr{D}^{\prime}\left(\mathbf{R}^{n}\right)$ given in [9]. But it seems that [9] contains more or less all those ideas which should be essential in proving a theorem analogous to Theorem 1 for a suitable class of (LF)-spaces of entire functions containing as particular examples the spaces of Fourier-Laplace transforms of (ultradifferentiable) test functions in an open convex subset of euclidean space.

\section{REFERENCES}

1. A. Baernstein II, Representation of holomorphic function by boundary integrals, Trans. Amer. Math. Soc. 160 (1971), 27-37.

2. C. A. Berenstein and M. A. Dostal, Analytically uniform spaces and their applications to convolution equations, Lecture Notes in Math., vol. 256, Springer-Verlag, Berlin and New York, 1972.

3. K.-D. Bierstedt, $A$ question on inductive limits of weighted locally convex function spaces, Atas do décimo primeiro colóquio brasileiro de matemática, Pocos de Caldas, Brasil, 1977; Vol. I, IMPA, Rio de Janeiro, 1978, pp. 213-226.

4. K.-D. Bierstedt, R. Meise and W. H. Summers, A projective description of weighted inductive limits, Paderborn, 1980 (preprint).

5. G. Björck, Linear partial differential operators and generalized distributions, Ark. Mat. 6 (1966), 351-407.

6. J.-E. Björk, The fundamental principle. Univ. of Stockholm, 1975 (preprint).

7. C.-C. Chou, La transformation de Fourier complexe et l'équation de convolution, Lecture Notes in Math., vol. 325, Springer-Verlag, Berlin and New York, 1973.

8. L. Ehrenpreis, A fundamental principle for systems of linear differential equations with constant coefficients, and some of its applications, Proc. Internat. Sympos. Linear Spaces (Jerusalem, 1960), Jerusalem Academic Press, Jerusalem; Pergamon, Oxford, 1971, pp. 161-174.

9. ___ Fourier analysis in several complex variables, Pure and Appl. Math., vol. 17, Wiley-Interscience, New York, 1970.

10. K. Floret and J. Wloka, Einführung in die Theorie der lokalkonvexen Räume, Lecture Notes in Math., vol. 56, Springer-Verlag, Berlin and New York, 1968.

11. L. Hörmander, Supports and singular supports of convolutions, Acta Math. 110 (1963), 279-302.

12. __ An introduction to complex analysis in several variables, 2nd ed., North-Holland, Amsterdam and London, 1973.

13. G. Köthe, Topologische lineare Räume. I, (2 Auflg.) Die Grundlehren der Math. Wissenschaften, Bd. 107, Springer-Verlag, Berlin, Heidelberg and New York, 1966. 
14. O. Liess, The fundamental principle of Ehrenpreis-Palamodov, Inst. of Math., Bucharest, 1976 (preprint).

15. Intersection properties of weak analytically uniform classes of functions, Ark. Mat. 16 (1976), 93-111.

16. V. I. Palamodov, Linear differential operators with constant coefficients, Die Grundlehren der Math. Wissenschaften, Bd. 168, Springer-Verlag, Berlin, Heidelberg and New York, 1979. English transl. of Linejuye differencial'nye operatory s postojannymi koefficientami, "Nauka", Moscow, 1967.

17. B. A. Taylor, A seminorm topology for some (DF)-spaces of entire functions. Duke Math. J. 38 (1971), 379-385.

MAthematik-Informatik, FB 17 der Gesamthochschule, D-4790 Paderborn, Federal Republic OF GERMANY 